\title{
Variations in the multimerization region of the Helicobacter pylori cytotoxin CagA affect virulence
}

\author{
DAIVA AHIRE, TRICIA ALSTON and ROBERT RAFFANIELLO
}

\begin{abstract}
Department of Medical Laboratory Sciences, School of Arts and Sciences, Hunter College, New York, NY 10010, USA
\end{abstract}
Received February 1, 2016; Accepted October 21, 2016

DOI: $10.3892 / 01.2017 .5562$

\begin{abstract}
Helicobacter pylori colonizes the human stomach by infecting gastric epithelial cells. It is the primary cause of peptic ulcer disease and gastric cancer (GC). Cytotoxin-associated gene $\mathrm{A}(\mathrm{CagA})$ is a virulence factor produced by $H$.pylori. Strains positive for the CagA protein are associated with more severe gastric diseases. The $3^{\prime}$ region of the cagA gene exhibits heterogeneity with respect to tyrosine phosphorylation motifs (EPIYA) and CagA multimerization motifs (CM). CagA proteins are categorized as either Western or Eastern based on EPIYA sequences. CM motifs are also identified as Western and Eastern based on CM sequences identified in Western and East Asian countries. It has been suggested that CagA proteins possessing an Eastern CM type are associated with less severe gastric disorders. In the present study, the effects of two CagA peptides with different CM motifs on cell function were compared: CagA with a Western and Eastern CM motif (CagA-WE), and CagA with two Western CM motifs (CagA-WW). CagA sequences were fused with green fluorescent protein (GFP) to form GFP-CagA fusion proteins. GFP-CagA and GFP control constructs were transfected into human gastric adenocarcinoma cells (AGS). GFP-CagA expression was verified by immunoblotting and immunofluorescence. The results demonstrated that, following $18 \mathrm{~h}$, the CagA-WE-transfected cells were less adherent compared with the CagA-WW transfected cells. CagA has also been reported to cause cell elongation in AGS cells. In the current study, cell elongation was more frequent in the CagA-WW-transfected cells compared with the CagA-WE transfected cells (8.34 vs. $3.97 \%$ cells, respectively). The CagA peptides did not affect proliferation or apoptosis rates. These results suggest that different $\mathrm{CM}$ motif types may affect CagA virulence.
\end{abstract}

Correspondence to: Dr Robert Raffaniello, Department of Medical Laboratory Sciences, School of Arts and Sciences, Hunter College, P.O. Box 617, 425 East 25th Street, New York, NY 10010, USA

E-mail: rraffani@hunter.cuny.edu

Key words: Helicobacter pylori, cytotoxin-associated gene A, gastric cancer, peptic ulcer, AGS cells, multimerization motifs

\section{Introduction}

Helicobacter pylori infection of the gastric mucosa results in gastritis in the majority of infected individuals (1). This infection is a major cause of peptic ulcer disease (PUD) and an important causative factor for gastric cancer (GC) (1). GC is the second most common cause of cancer-associated mortalities and is one of the most prevalent malignancies worldwide (2). H. pylori-induced progression towards PUD or GC is likely to depend upon a combination of several factors, including the bacterial genotype, host susceptibility, immune response and environmental factors.

Cytotoxin-associated gene A (CagA) is a $120-145-\mathrm{kDa}$ protein produced by $H$. pylori and is the product of the $c a g A$ gene $(3,4)$. The cagA gene is not present in all H.pylori strains and the majority of studies report an increased risk of PUD and GC with CagA-positive H. pylori strains $(5,6)$. In patients infected with CagA-positive H. pylori strains, the CagA cytotoxin is injected into the epithelial cells of the stomach. The C-terminal region of CagA varies among $H$. pylori strains and is the target region for tyrosine phosphorylation by host cell kinases (7). Four tyrosine phosphorylation motifs (EPIYA) are located within this region and have been characterized with respect to the amino acid sequences flanking these sites $(7,8)$. These four sites, termed EPIYA-A, -B, -C and -D, vary in number and organization in different $H$. pylori strains $(7,8)$. Based on the arrangement of the EPIYA motifs, there are two types of CagA designated Western CagA and East Asian CagA (8). The Western type CagA has EPIYA-A and EPIYA-B sites followed by 1 to 3 EPIYA-C sites, while the East Asian CagA has EPIYA-A and EPIYA-B sites, but lacks EPIYA-C (8). The third domain of the East Asian CagA is EPIYA-D (8). Regarding clinical significance, the East Asian $\mathrm{CagA}$ is considered to be more virulent than the Western type. For the Western CagA, an increased number of EPIYA-C motifs is associated with greater pathogenicity (8).

Within the variable region of the $\operatorname{cagA}$ gene is another motif called the CagA multimerization (CM) region (9). This is a 16-amino acid sequence, which is responsible for dimerization of CagA (9). Similar to the EPIYA patterns, Western and Eastern CM sequences have been identified (10). However, unlike CagA EPIYA motifs, the frequency and arrangement of Western and Eastern CM motifs and their association with clinical outcomes has not been well studied. In Western CagA proteins, a CM motif is often located prior to and following 
the EPIYA-C motif. A previous study examined H. pylori infection in a minority patient population in New York City and observed that this population had a higher infection rate than the general population (11). Furthermore, this population is reportedly infected with more virulent CagA-positive $H$. pylori strains that are associated with an increased risk of precancerous changes in the gastric mucosa (12).

A subsequent study examined cagA genes from $H$. pylori strains infecting the same patient population from New York City (13). It was reported that all CagA proteins were of the Western type with respect to EPIYA motifs and the majority of the EPIYA motifs were arranged in an ABC pattern (13) However, a greater degree of heterogeneity in the samples with respect to the CM motifs surrounding the EPIYA-C phosphorylation motif was observed (13). Notably, analysis of the CM types and arrangements in these samples suggests that the presence of one or two Western CM motifs (designated WW or W-) in the absence of an Eastern CM motif is associated with more severe gastric disorders like PUD and GC (13). Consistent with these results, $H$. pylori strains expressing Western CagA proteins with the Western/Western CM motif pattern have been associated with a greater mean length of cell elongation and a greater affinity for SHP-2 when compared with CagA proteins with the Western/Eastern pattern (10). This suggests that in Western CagA proteins, which contain the ABC EPIYA pattern, an Eastern CM motif may decrease CagA multimerization and virulence. Therefore, in $H$.pylori-infected patients that are $\operatorname{cag} A$-positive, the pattern and types of CM motifs may be clinically significant.

Although the CM regions may affect the magnitude of the pathophysiological activities of CagA, the effect of particular $\mathrm{CM}$ sequences and arrangements on cell function has not yet been studied. The present study focused specifically on the effects of CagA proteins with Western/Western and Western/Eastern CM patterns on gastric cell function using human gastric adenocarcinoma (AGS) cells, a human gastric tumor cell line. The results suggest that different CM motif types and arrangements may affect CagA virulence.

\section{Materials and methods}

Cloning and mutagenesis of the CagA $C$-terminal region. The C-terminal region of Western CagA proteins usually contain at least three EPIYA motifs (EPIYA-A, EPIYA-B and EPIYA-C), in addition to two CM motifs flanking the EPIYA-C motif (7-9). The CagA C-terminal region obtained from a $H$. pylori-infected subject from a previous study (13) was cloned and contained the terminal 386 amino acids of the CagA protein (based on CagA sequence CBV36576). This CagA C-terminal fragment was amplified by polymerase chain reaction and cloned into the pAcGFP1-C3 vector (Clontech Laboratories, Inc., Mountainview, CA, USA) downstream to and in frame with green fluorescent protein (GFP). The primers used were as follows: CagA-DF, GGATTGAGCTCA TGGGAACAGGCGATTTCAGTGGGGTAG and CagA-DR, CCGAGGTACCTTAAGATTTTTGGAAACCACCTTTTG

TA (14). The CagA-DF and CagA-DR primers contained SacI and $K p n$ I restriction sites (italicized above), respectively.

The portion of the cloned peptide containing the EPIYA and $\mathrm{CM}$ motifs is presented in Fig. 1A and contains three
EPIYA phosphorylation motifs (A, B and C), in addition to Western and Eastern CM motifs flanking the EPIYA-C region. The amino acid sequence of the underlined portion of the Eastern CM motif (Fig. 1A) was mutated from RRS to $\mathrm{KKH}$, and subsequently resembled a Western CM motif based on CM motifs observed in strains from Eastern or Western countries (10). The sequence integrity of each construct was verified by DNA sequencing. The result was two GFP-CagA constructs that were identical except for the CM motifs flanking the EPIYA-C region (Fig. 1B). One construct, GFP-CagA-WE, contained a Western/Eastern CM motif arrangement, while the other, GFP-CagA-WW, contained a Western/Western arrangement.

Cell culture and transfection. AGS cells were obtained from the American Type Culture Collection (Manassas, VA, USA) and cultured in F12K media with $10 \%$ fetal bovine serum (Hyclone; GE Healthcare Life Sciences, Logan, UT, USA), $1 \mathrm{X}$ penstrep and $2 \mathrm{mM}$ glutamine. Cells were grown on T-25 flasks, 6-well plates or Nunc chamber slides and transfected using Lipofectamine ${ }^{\circledR} 2000$ (Thermo Fisher Scientific, Inc., Waltham, MA, USA) according to manufacturer's protocol. The DNA to Lipofectamine ratio was 1:2.5 and transfection efficiency was $>75 \%$. Cell viability was measured using the Trypan blue (TB) exclusion assay. Briefly, equal amounts of cell suspension were mixed with $0.4 \%$ TB in PBS. The cells were examined under a microscope and the percentage of TB-positive cells was determined.

Immunoblotting of subcellular fractions. AGS cells were transfected with plasmids containing the GFP-CagA constructs, GFP without CagA or no plasmid. After 18-20 h, the cells were washed with PBS and harvested by scraping into ice-cold PBS and centrifuged at $1,500 \times \mathrm{g}$ for $5 \mathrm{~min}$ at room temperature. In certain experiments, detached cells in the media were collected by centrifugation at $1,500 \times \mathrm{g}$ for 5 min at room temperature and washed with PBS. Cell pellets were resuspended in lysing solution [20 $\mathrm{mM}$ Tris-HCL (pH 7.2), $150 \mathrm{mM} \mathrm{NaCl}, 1 \%$ TX-100, 1 mM NaF, 2 mM $\mathrm{Na}_{3} \mathrm{VO}_{4}$ and 1X Halt protease inhibitor cocktail (Thermo Fisher Scientific, Inc.)]. Protein assays were performed using the Coomassie Protein assay reagent (Thermo Fisher Scientific, Inc.) according to the manufacturer's protocol, and immunoblotting was performed as previously described (15). Primary antibodies included anti-GFP (\#PA1980A; Thermo Fisher Scientific, Inc.), anti-Caspase-3 (\#9662; Cell Signaling Technology, Inc., Danvers, MA, USA) and Ki-67 (\#RB9043; Thermo Fisher Scientific, Inc.) and were diluted at 1:1,000.

Immunocytochemistry. Cells were grown on Nunc chamber slides and transfected as aforementioned. Following $18 \mathrm{~h}$, the cells were fixed with $4 \%$ paraformaldehyde for $10 \mathrm{~min}$ and washed in PBS. GFP-CagA proteins were visualized by immunofluorescence. For Ki-67 staining, the cells were incubated with PBS containing 3\% bovine serum albumin (BSA), $1 \%$ goat serum (Thermo Fisher Scientific, Inc.) and 0.2\% Triton X-100 (PBS-BSA/GS) for $1 \mathrm{~h}$ followed by incubation with anti-Ki-67 $(1: 1,000)$ overnight at $4^{\circ} \mathrm{C}$. The slides were incubated with Alexa Fluor-568-conjugated goat anti-rabbit immunoglobulin G secondary antibodies, washed and mounted with DAPI 
A

\section{CagA-WE}

NFNNNSNGLKNGKDKGPEEPIYAQVNKKKTGHVASPEEPIYAQVAKKVTK KIDQLNQAATSGFGGVGQAGFPLKKHANIEDLSKVGRSVSPEPIYATIDD LGGSFPLRRSAKVEDLSKVGLQGIKN

\section{CagA-WW}

NFNNNSNGLKNGKDKGPEEPIYAQVNKKKTGHVASPEEPIYAQVAKKVTK KIDQLNQAATSGFGGVGQAGFPLKKHANIEDLSKVGRSVSPEPIYATIDD LGGSFPLKKHAKVEDLSKVGLQGIKN
B

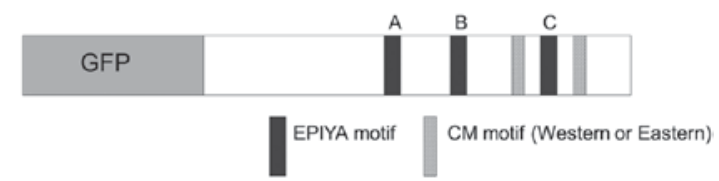

Figure 1. CagA C-terminal regions used in the present study. (A) A CagA peptide fragment containing the C-terminal 386 amino acids was cloned from a Helicobacter pylori strain expressing the W Type CagA. The portion of the peptide that contains the EPIYA phosphorylation motifs and the CM regions are presented. The EPIYA motifs and CM regions are in bold. This peptide, termed as CagA-WE, contains two 16 amino acid CM motifs that flank the third EPIYA motif of the original sequence. CagA-WE contains a W and E CM region, respectively. CagA-WW was obtained by mutating the three underlined amino acids from RRS to KKH in the second CM motif of CagA-WE (underlined). (B) Schematic of the constructs used in the present study. The CagA C-terminal peptides were cloned downstream and in frame with GFP in the pAcGFP1-C3 vector. The EPIYA and CM motifs are presented. CagA, cytotoxin-associated gene A; W, Western; EPIYA, tyrosine phosphorylation motifs; CM, CagA multimerization; E, Eastern; GFP, green fluorescent protein.

containing mounting media. Fluorescent signals were visualized using an SP2 confocal microscope (Leica Microsystems, Inc., Buffalo Grove, IL, USA) or an AX10 microscope (Zeiss AG, Oberkochen, Germany). Images were obtained, merged and assembled using Adobe Photoshop software v7.0 (Adobe Systems, Inc., San Jose, CA, USA). To measure apoptosis in transfected cells, the In Situ Cell Death Detection kit (Roche, Indianapolis, IN, USA) was used according to the manufacturer's protocol.

Cell elongation assays. CagA has been reported to induce cell elongation or the 'hummingbird phenotype' in epithelial cells $(16,17)$. Hummingbird cells are defined as having needle-like cellular protrusions $>5 \mu \mathrm{m}$ in length. To determine whether or not the CM motif type and arrangement affected AGS cell morphology, the percent of transfected cells exhibiting this phenotype was determined. At least 200 cells were counted per transfectant in each experiment. The average length of the cell protrusions of at least 50 cells per transfectant was measured using Spot Advanced software v4.6 (SPOT Imaging Solutions; Diagnostic Instruments, Inc., Sterling Heights, MI, USA) on images of captured with an AX10 microscope (Zeiss AG).

Statistical analysis. Data presented in the figures are representative of at least three different experiments. The data are presented as the mean \pm standard error of the mean. Comparisons between groups were evaluated by Student's $t$-test, and $\mathrm{P}<0.05$ were considered to indicate a statistically significant difference.

\section{Results and Discussion}

CagA constructs and expression in AGS cells. In the present study, the effects of two CagA C-terminal peptides with two different $\mathrm{CM}$ patterns on epithelial function and morphology were examined. The only difference between the two CagA constructs used in the current study was the CM pattern. These constructs were cloned downstream and in frame with GFP as described in the Materials and methods section and are presented in Fig. 1A and B. The CagA C-terminal fragment containing two Western type $\mathrm{CM}$ regions is referred to as the CagA-WW, while the fragment containing Western and Eastern CM motifs is referred to as the CagA-WE. The vector containing GFP without a CagA fragment was used as a control. Previous studies have demonstrated that the C-terminal CagA fragment exhibits CagA activity, including the induction of cell elongation and interleukin- 8 secretion in AGS cells $(14,18)$. This fragment, which contains the terminal third of the CagA protein, was observed to induce greater cell elongation than the complete CagA protein (18).

AGS cells were transfected with plasmids coding for GFP, GFP-CagA-WE, GFP-CagA-WW or no plasmid. After $18 \mathrm{~h}$, lysates were prepared and immunoblotted with an anti-GFP antibody. As presented in Fig. 2A (lane 1), a 27-kD band was observed in lysates from cells transfected with the GFP plasmid, which corresponds with the molecular weight (MW) of GFP. In cells transfected with no plasmid, no new bands were observed (Fig. 2A, lane 4). By contrast, an $\sim 70-\mathrm{kD}$ band was observed in lysates prepared from cells expressing either GFP-CagA-WW or GFP-CagA-WE (Fig. 2A, lanes 2 and 3, respectively). The 70-kD band was observed just above a non-specific band present in all four samples. Three additional low MW bands were observed in lysates prepared from cells expressing either GFP-CagA-WW or GFP-CagA-WE at $\sim 55 \mathrm{kDa}, 40 \mathrm{kDa}$ and $35 \mathrm{kDa}$ in lanes 2 and 3 (Fig. 2A). These may represent cleaved fragments of the GFP-CagA peptide. Fragmentation of GFP-CagA was observed to varying degrees in cell lysates from different transfection experiments (Fig. 2B and C). The level of expression of GFP-CagA-WW and GFP-CagA-WE was similar in cells transfected with these plasmids.

Cleavage of CagA following infection of epithelial cells with $H$. pylori has been observed previously and it was suggested that CagA cleavage may be involved with host signal transduction and virulence $(19,20)$. Notably, CagA cleavage is not observed in bacterial cells, and occurs only following translocation into the host cell (20). It is unlikely that cleavage of the GFP-CagA protein occurred as a result of cell manipulation as lysates were prepared in buffers containing protease and phosphatase inhibitors and cold reagents, and, as aforementioned, the GFP remained intact. Whether or not CagA requires fragmentation to function remains to be determined.

In the current study, attempts were made to use a commercially prepared CagA-specific antibody to immunoblot lysates prepared from GFP-CagA expressing cells. However, this antibody did not recognize the GFP-CagA proteins. This was 

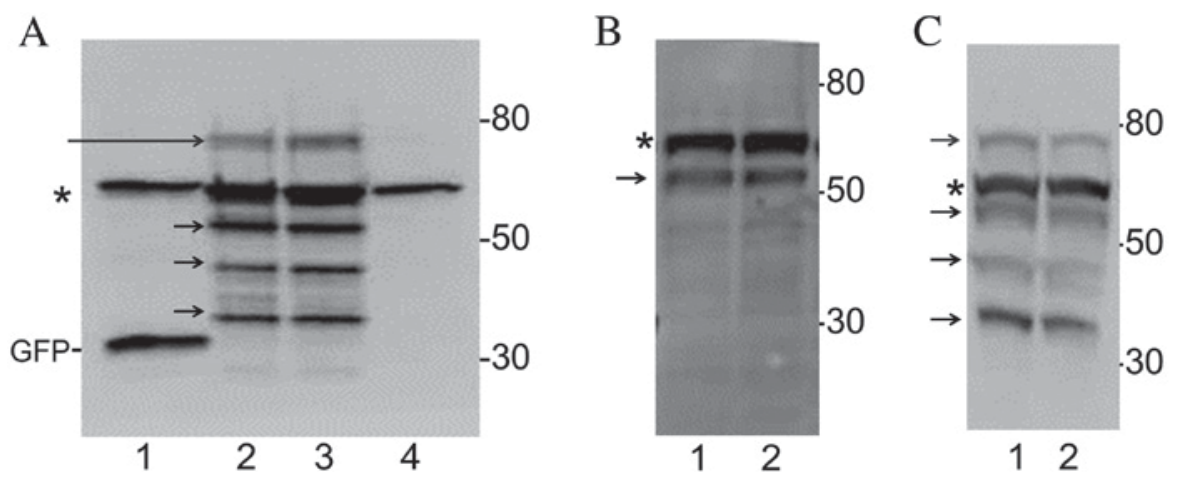

Figure 2. Immunoblotting of GFP-CagA proteins in transfected AGS cells. (A) AGS cells were transfected with plasmids containing (Lane 1) GFP alone, (Lane 2) GFP-CagA-WE, (Lane 3) GFP-CagA-WW or (Lane 4) no plasmid. Lysates were prepared and immunoblotted as described. The asterisk indicates a non-specific protein that was present in all samples. Long arrow indicates full length GFP-CagA peptide and short arrows indicate GFP-CagA peptide fragments. (B and C) Immunoblots of lysates prepared from (Lane 1) GFP-CagA-WE or (Lane 2) GFP-CagA-WW expressing AGS cells from other experiments. Molecular weight markers are on the right. CagA, cytotoxin-associated gene A; GFP, green fluorescent protein; AGS, human gastric adenocarcinoma cells; W, Western; E, Eastern.

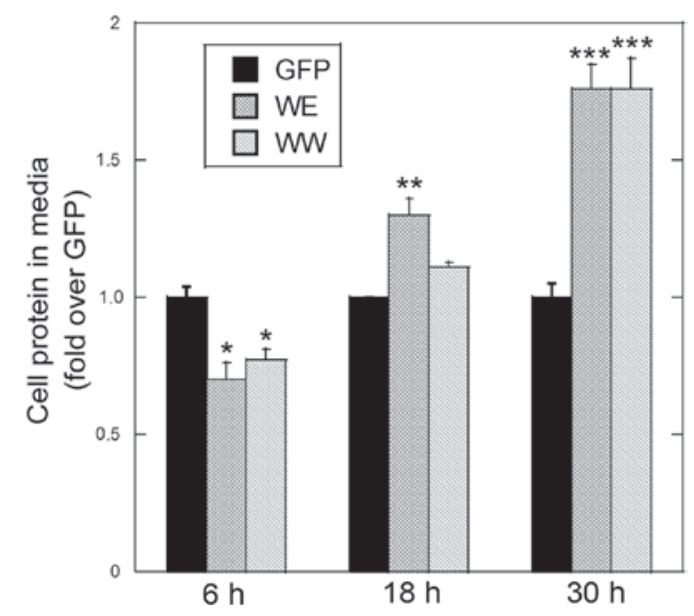

Figure 3. Effect of CagA constructs on AGS cell adherence. AGS cells were grown on chamber slides and transfected with plasmids containing GFP alone, GFP-CagA-WE or GFP-CagA-WW. After 6, 18 or 30 h, cellular protein levels in adherent and detached cells were determined as described. The percent of total cellular protein in the media (detached cells) was determined. Data were normalized to percent of total cellular protein in the media in GFP-expressing cells and plotted. Error bars represent standard error of the mean. "Significantly less than GFP alone $(\mathrm{P}<0.05) ;{ }^{* *}$ significantly greater than WW $(\mathrm{P}<0.05)$; and ${ }^{* * *}$ significantly greater than GFP alone $(\mathrm{P}<0.05)$ CagA, cytotoxin-associated gene A; AGS, human gastric adenocarcinoma cells; GFP, green fluorescent protein; W, Western; E, Eastern.

most likely due to the fact that the C-terminal is the most variable region in the CagA protein and may not be recognized by commercially prepared antibodies. Lack of antigenicity in cells expressing the CagA C-terminal fragment has been observed previously (14). Nevertheless, the sequences of each GFP-CagA construct were analyzed and it was determined that the CagA sequences were in frame with the GFP sequence in the plasmid. Furthermore, the size of the high MW band recognized by the GFP antibody was consistent with the calculated MW of the GFP-CagA constructs, which was $\sim 69 \mathrm{kD}$.

CagA expression affects AGS cell adhesion. In the present study, an apparent increase in detached cells in AGS cells expressing either GFP-CagA-WE or GFP-CagA-WW for $18 \mathrm{~h}$ or longer was observed. To quantitate the effect of CagA on cell adhesion, the cells were transfected and grown for 6, 18 or $30 \mathrm{~h}$. The protein levels of adherent and detached cells were determined and expressed as percent cellular protein in media. The detached cells were washed in PBS to rid the sample of secreted proteins or proteins derived from the FBS added to the media. The values were normalized to the protein level in detached cells transfected with the GFP plasmid. At $6 \mathrm{~h}$ post-transfection, a lower level of cellular protein in the media was observed with cells expressing either GFP-CagA-WE or GFP-CagA-WW compared with cells expressing GFP alone (Fig. 3). This suggests that CagA-transfected cells were more adherent at this early time point. At $18 \mathrm{~h}$ post-transfection, a higher level of protein in the media was observed with cells expressing either GFP-CagA-WE or GFP-CagA-WW compared with cells expressing GFP alone (Fig. 3). Furthermore, at this time point the amount of cellular protein in the media was higher in GFP-CagA-WE expressing cells compared with GFP-CagA-WW expressing cells (Fig. 3). At $36 \mathrm{~h}$ post-transfection, protein levels in the media were almost two-fold higher in cells expressing either GFP-CagA-WE or GFP-CagA-WW compared with cells expressing GFP alone (Fig. 3). The viability of the detached cells was $>85 \%$ in all cases indicating that these are not cells that died and detached from the culture surface. These results suggest that different $\mathrm{CM}$ motif patterns affect the rate at which the cells attach to the surface and that each form of CagA inhibits cell attachment at time points beyond $18 \mathrm{~h}$.

Other researchers have also observed decreased cell adhesion in cells transfected with CagA or infected with $\mathrm{CagA}^{+} H$. pylori strains (21). This is not remarkable since it has been demonstrated that $H$. pylori infection with $\mathrm{CagA}^{+}$ strains perturbs various cell junction types, including tight junctions $(22,23)$, focal adhesion complexes $(24,25)$ and gap junctions (26). The results of the current study indicate that overexpression of CagA eventually inhibits cell attachment and that the effect of CagA on cell attachment is time-dependent. Additionally, CagA-WE appears to inhibit cell attachment sooner (18 h post-transfection). Further studies are required to determine how the different CagA $\mathrm{CM}$ regions affect specific cell junction types and how this relates to gastric pathology. 


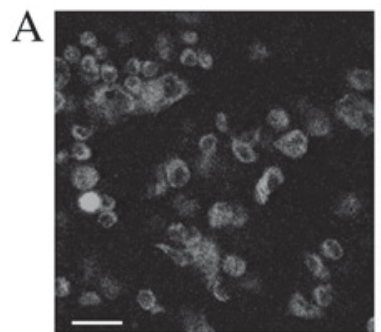

GFP

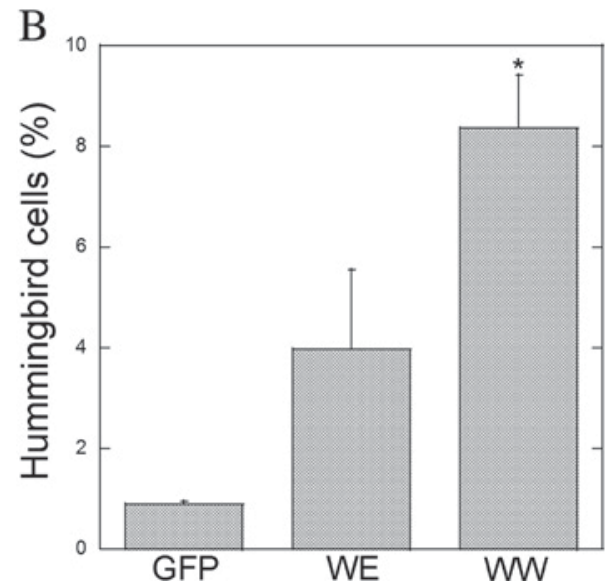

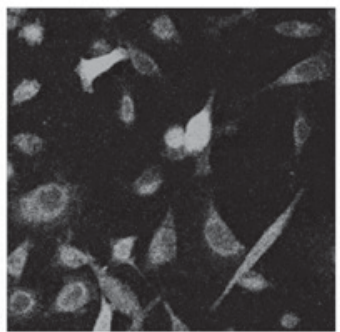

WE

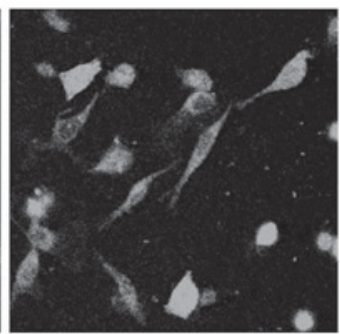

WW

$\mathrm{C}$

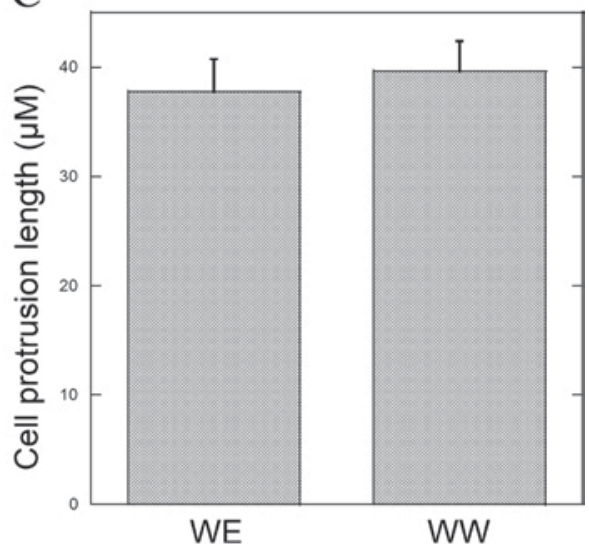

Figure 4. GFP and GFP-CagA expression and localization in AGS cells. (A) Confocal microscopy. AGS cells were grown on chamber slides, transfected and prepared as described. GFP immunofluorescence was observed by confocal microscopy. Note the diffuse fluorescence staining pattern in all micrographs (scale bar, $20 \mu \mathrm{M}$ ). (B) Number of elongated cells in sample of transfected AGS cells. Elongated cells were counted in random fields in groups of cells transfected with GFP, GFP-CagA-WE or GFP-CagA-WW. The percent of elongated cells was determined. (C) Protrusion length in transfected AGS cells. The average length of the cell protrusions of at least 50 cells in GFP-CagA-WE or GFP-CagA-WW transfected cells was measured as described. Error bars represent the standard error of the mean. *Significantly greater than WE (P<0.05). GFP, green fluorescent protein; CagA, cytotoxin-associated gene A; AGS, human gastric adenocarcinoma cells; W, Western; E, Eastern.
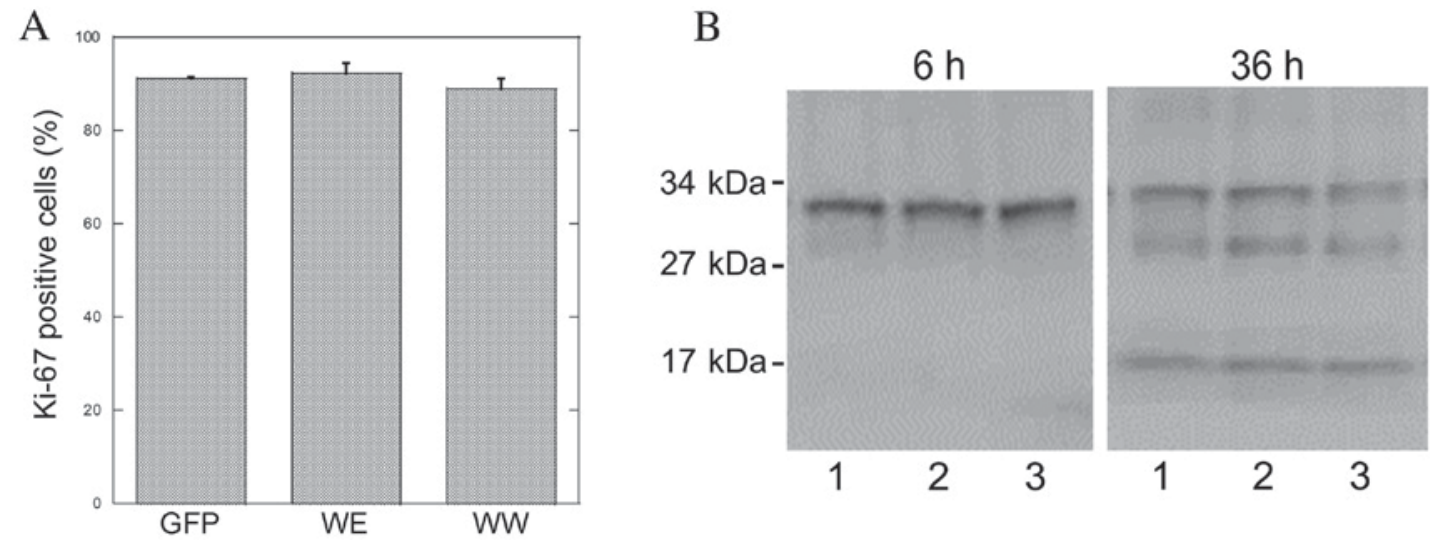

Figure 5. Effect of CagA constructs on AGS cell proliferation and apoptosis. (A) Ki-67 staining. AGS cells were grown on chamber slides and transfected with plasmids containing GFP alone, GFP-CagA-WE or GFP-CagA-WW. The cells were stained for Ki-67, a marker of cell proliferation. Ki-67-positive cells were counted and the percentage was determined. (B) Immunoblotting of Caspase-3. AGS cells were transfected with GFP, GFP-CagA-WE or GFP-CagA-WW containing plasmids. Cell lysates were prepared at 6, 18 and $36 \mathrm{~h}$. Lysates were immunoblotted for Caspase-3 as described in Materials and methods. CagA, cytotoxin-associated gene A; AGS, human gastric adenocarcinoma cells; W, Western; E, Eastern.

Effect of GFP-CagA on cell elongation. Cell elongation or the 'hummingbird' phenotype is a well-accepted in vitro measure of CagA virulence and is associated with the ability of CagA to interact with SHP-2 $(17,19)$. To examine the effects of the different CM motifs on cell elongation, AGS cells were transfected with GFP, GFP-CagA-WE or GFP-CagA-WW containing plasmids. Following $18 \mathrm{~h}$, the cells were fixed and mounted. When examined at higher power by confocal microscopy, the appearance of needle-like cytoplasmic projections was more apparent in GFP-CagA expressing cells (Fig. 4A). In GFP-expressing cells, the staining pattern was cytoplasmic. By contrast, the staining appeared to be cytoplasmic and nuclear in cells expressing GFP-CagA-WE or GFP-CagA-WW. Note that the staining intensity of the green fluorescence appeared to be similar in cells transfected with the GFP, CagA-WE and CagA-WW plasmids. 
The percentage of elongated cells in transfected AGS cells was determined. A higher percentage of elongated cells was observed with either GFP-CagA construct compared with cells expressing GFP alone (Fig. 4B). Notably, the highest percentage of elongated cells was observed in cells expressing GFP-CagA-WW (Fig. 4B). However, the average length of the cell protrusions was not different in GFP-CagA-WE or GFP-CagA-WW expressing cells (Fig. 4C).

Taken together, these results suggest that the WW CM motif pattern is more virulent than the WE pattern, which is consistent with the observations of a previous study (10). However, in this previous study, various CagA proteins were used that differed in EPIYA motifs and other portions of the CagA sequence, whereas the results of the present study may be attributed solely to variations in the $\mathrm{CM}$ region as this was the only variable region in the CagA constructs. Therefore, to the best of our knowledge, the current study has demonstrated for the first time that the CM pattern affects the ability of CagA to induce cell elongation.

Effect of GFP-CagA on cell proliferation and apoptosis. The effect of CagA protein expression on cell proliferation in AGS cells was measured using Ki-67 antibodies as an indicator of cell proliferation. The level of labeling with Ki-67 was $~ 90 \%$ and did not vary in GFP, GFP-CagA-WE or GFP-CagA-WW expressing AGS cells (Fig. 5A). Few apoptotic cells were detected using the TUNEL assay and due to the extremely low level of apoptotic activity, accurate apoptotic measurements could not be obtained (data not shown). Nevertheless, the level of apoptosis as measured by the TUNEL assay did not appear different in GFP-CagA-WE or GFP-CagA-WW expressing cells. Apoptotic activity was also examined in transfected AGS cells by immunoblotting with the caspase-3 antibody. The cleaved or active form of caspase-3 (17 kDa) was not present in transfected cells at 6 or $18 \mathrm{~h}$ post-transfection (Fig. 5B). By contrast, the caspase- 3 fragment was visible $36 \mathrm{~h}$ post-transfection in GFP, GFP-CagA-WE or GFP-CagA-WW expressing cells (Fig. 5B). However, the levels of the cleaved caspase-3 fragment were not different in cells transfected with the GFP- or CagA-containing plasmids indicating that expression of the CagA peptide does not affect apoptotic rates under these conditions.

Depending on the type of assay, H. pylori infection has been associated with increased or decreased proliferation (27-29). The CagA constructs utilized in the present study did not affect the rate of proliferation or apoptosis in AGS cells regardless of the $\mathrm{CM}$ pattern. It is possible that these effects require long-term expression of the $\mathrm{Cag} \mathrm{A}$ protein, which would be observed in chronically infected tissue, or that other $H$. pylori proteins are required to affect proliferation and/or apoptosis.

In conclusion, to the best of our knowledge, the current study demonstrated for the first time that heterogeneity in the CM motif patterns of the CagA virulence factor affects cell adhesion and morphology. Further studies are required to elucidate the mechanisms underlying these effects. Furthermore, as there is a significant degree of heterogeneity in $\mathrm{CM}$ motifs from different $H$. pylori strains, closer investigation into how these differences affect $\mathrm{Cag} \mathrm{A}$ virulence and clinical outcomes is warranted.

\section{Acknowledgements}

The present study was supported in part by grants from PSC-CUNY, New York, NY, USA (no. 64464-0042), The Hunter College Presidential Fund for Faculty Advancement, New York, NY, USA (no. 94806-6013) and The Shuster Faculty Fellowship Fund, New York, NY, USA (no. 78742).

\section{References}

1. Qadri Q, Rasool R, Gulzar GM, Naqash S and Shah ZA: H pylori infection, inflammation and gastric cancer. J Gastrointest Cancer 45: 126-132, 2014.

2. Torre LA, Bray F, Siegel RL, Ferlay J, Lortet-Tieulent J and Jemal A: Global cancer statistics, 2012. CA Cancer J Clin 65: 87-108, 2015.

3. Covacci A, Censini S, Bugnoli M, Petracca R, Burroni D, Macchia G, Massone A, Papini E, Xiang Z and Figura N: Molecular characterization of the $128-\mathrm{kDa}$ immunodominant antigen of Helicobacter pylori associated with cytotoxicity and duodenal ulcer. Proc Natl Acad Sci USA 90: 5791-5795, 1993.

4. Tummuru MK, Cover TL and Blaser MJ: Cloning and expression of a high-molecular-mass major antigen of Helicobacter pylori: Evidence of linkage to cytotoxin production. Infect Immun 61: 1799-1809, 1993

5. Brenner H, Arndt V, Stegmaier C, Ziegler H and Rothenbacher D: Is Helicobacter pylori infection a necessary condition for noncardia gastric cancer? Am J Epidemiol 159: 252-258, 2004.

6. Hatakeyama M and Higashi H: Helicobacter pylori CagA: A new paradigm for bacterial carcinogenesis. Cancer Sci 96: 835-843, 2005.

7. Stein M, Bagnoli F, Halenbeck R, Rappuoli R, Fantl WJ and Covacci A: c-Src/Lyn kinases activate Helicobacter pylori CagA through tyrosine phosphorylation of the EPIYA motifs. Mol Microbiol 43: 971-980, 2002.

8. Higashi H, Tsutsumi R, Fujita A, Yamazaki S, Asaka M, Azuma T and Hatakeyama M: Biological activity of the Helicobacter pylori virulence factor CagA is determined by variation in the tyrosine phosphorylation sites. Proc Natl Acad Sci USA 99: 14428-14433, 2002.

9. Ren S, Higashi H, Lu H, Azuma T and Hatakeyama M: Structural basis and functional consequence of Helicobacter pylori CagA multimerization in cells. J Biol Chem 281: 32344-32352, 2006.

10. Sicinschi LA, Correa P, Peek RM, Camargo MC, Piazuelo MB, Romero-Gallo J, Hobbs SS, Krishna U, Delgado A, Mera R, et al: CagA C-terminal variations in Helicobacter pylori strains from Colombian patients with gastric precancerous lesions. Clin Microbiol Infect 16: 369-378, 2010.

11. Straus EW, Patel H, Chang J, Gupta RM, Sottile V, Scirica J, Tarabay G, Iyer S, Samuel S and Raffaniello RD: H. pylori infection and genotyping in patients undergoing upper endoscopy at inner city hospitals. Dig Dis Sci 47: 1575-1581, 2002.

12. Schneller J, Gupta R, Mustafa J, Villanueva R, Straus EW and Raffaniello RD: Helicobacter pylori infection is associated with a high incidence of intestinal metaplasia in the gastric mucosa of patients at inner-city hospitals in New York. Dig Dis Sci 51: 1801-1809, 2006.

13. Ogorodnik E and Raffaniello RD: Analysis of the 3'-variable region of the cagA gene from Helicobacter pylori strains infecting patients at New York City hospitals. Microb Pathog 56: 29-34, 2013.

14. Kim SY, Lee YC, Kim HK and Blaser MJ: Helicobacter pylori CagA transfection of gastric epithelial cells induces interleukin-8. Cell Microbiol 8: 97-106, 2006.

15. Limi S, Ojakian G and Raffaniello R: Rab3D regulates amylase levels, not agonist-induced amylase release, in AR42 J cells. Cell Mol Biol Lett 17: 258-273, 2012.

16. Higashi H, Nakaya A, Tsutsumi R, Yokoyama K, Fujii Y, Ishikawa S, Higuchi M, Takahashi A, Kurashima Y, Teishikata Y, et al: Helicobacter pylori CagA induces Ras-independent morphogenetic response through SHP-2 recruitment and activation. J Biol Chem 279: 17205-17216, 2004.

17. Segal ED, Cha J, Lo J, Falkow S and Tompkins LS: Altered states: Involvement of phosphorylated CagA in the induction of host cellular growth changes by Helicobacter pylori. Proc Natl Acad Sci USA 96: 14559-14564, 1999. 
18. Pelz C, Steininger S, Weiss C, Coscia F and Vogelmann R: A novel inhibitory domain of Helicobacter pylori protein CagA reduces CagA effects on host cell biology. J Biol Chem 286: 8999-9008, 2011.

19. Backert S, Muller EC, Jungblut PR and Meyer TF: Tyrosine phosphorylation patterns and size modification of the Helicobacter pylori CagA protein after translocation into gastric epithelial cells. Proteomics 1: 608-617, 2001

20. Moese S, Selbach M,Zimny-Arndt U, Jungblut PR, Meyer TF and Backert S: Identification of a tyrosine-phosphorylated $35 \mathrm{kDa}$ carboxy-terminal fragment (p35CagA) of the Helicobacter pylori CagA protein in phagocytic cells: Processing or breakage? Proteomics 1: 618-629, 2001.

21. Alfizah $\mathrm{H}$ and Ramelah M: Variant of Helicobacter pylori CagA proteins induce different magnitude of morphological changes in gastric epithelial cells. Malays J Pathol 34: 29-34, 2012.

22. Amieva MR, Vogelmann R, Covacci A, Tompkins LS, Nelson WJ and Falkow S: Disruption of the epithelial apical-junctional complex by Helicobacter pylori CagA. Science 300: 1430-1434, 2003 .

23. Song X, Chen HX, Wang XY, Deng XY, Xi YX, He Q, Peng TL, Chen J, Chen W, Wong BC and Chen MH: H. pylori-encoded CagA disrupts tight junctions and induces invasiveness of AGS gastric carcinoma cells via Cdx2-dependent targeting of Claudin-2. Cell Immunol 286: 22-30, 2013.
24. Moese S, Selbach M, Brinkmann V, Karlas A, Haimovich B, Backert S and Meyer TF: The Helicobacter pylori CagA protein disrupts matrix adhesion of gastric epithelial cells by dephosphorylation of vinculin. Cell Microbiol 9: 1148-1161, 2007.

25. Schneider S, Weydig C and Wessler S: Targeting focal adhesions: Helicobacter pylori-host communication in cell migration. Cell Commun Signal 6: 2, 2008.

26. Tao R, Hu MF, Lou JT and Lei YL: Effects of H. pylori infection on gap-junctional intercellular communication and proliferation of gastric epithelial cells in vitro. World J Gastroenterol 13: 5497-5500, 2007.

27. Peek RM Jr, Moss SF, Tham KT, Pérez-Pérez GI, Wang S, Miller GG, Atherton JC, Holt PR and Blaser MJ: Helicobacter pylori cagA+ strains and dissociation of gastric epithelial cell proliferation from apoptosis. J Natl Cancer Inst 89: 863-868, 1997.

28. Ricci V, Ciacci C, Zarrilli R, Sommi P, Tummuru MK, Del Vecchio Blanco C, Bruni CB, Cover TL, Blaser MJ and Romano M: Effect of Helicobacter pylori on gastric epithelial cell migration and proliferation in vitro: Role of VacA and CagA. Infect Immun 64: 2829-2833, 1996.

29. Rudnicka W, Covacci A, Wadstrom T and Chmiela M: A recombinant fragment of Helicobacter pylori CagA affects proliferation of human cells. J Physiol Pharmacol 49: 111-119, 1998. 